\title{
Einstein-Hilbert action on the brane for the bulk graviton
}

\author{
Steven Corley, ${ }^{*}$ David A. Lowe ${ }^{\dagger}$ and Sanjaye Ramgoolam ${ }^{\ddagger}$ \\ Department of Physics \\ Brown University \\ Providence, RI 02912 \\ USA
}

\begin{abstract}
We find new closed string couplings on Dp-branes for the bosonic string. These couplings are quadratic in derivatives and therefore take the form of induced kinetic terms on the brane. For the graviton in particular we find the induced Einstein-Hilbert term as well as terms quadratic in the second fundamental tensor. We comment on tachyon dependences of these brane-localized couplings.
\end{abstract}

*scorley@het. brown.edu

†lowe@het. brown.edu

${ }^{\ddagger}$ ramgosk@het. brown. edu 


\section{Contents}

1. Introduction 1

2. String scattering amplitudes 3

3. Comparing to the field theory limit 6

3.1 Graviton scattering at $\mathcal{O}\left(\left(\alpha^{\prime}\right)^{0}\right)$

3.2 Graviton scattering at $\mathcal{O}\left(\alpha^{\prime}\right)$

3.3 Dilaton and Antisymmetric Tensor scattering [1]

4. Field redefinition ambiguities 13

5. Scattering of brane-localized fields and the Einstein-Hilbert term

6. Comments on tachyon terms on the brane 17

A. The first and second fundamental tensors 18

\section{Introduction}

$D$-branes have played a prominent role in string theory since the realization that they are the carriers of Ramond-Ramond (or RR) charge arising in the superstring. They are described in the sigma-model context by submanifolds where open strings end. From the spacetime point of view, and at low energies compared to the string scale, they are submanifolds on which massless fields propagate and interact with the bulk, or closed string, fields. To leading order in an $\alpha^{\prime}$ expansion with respect to the closed string fields (to zeroth order in derivatives on the closed string fields) the low energy effective action governing the $D$-brane - bulk interactions for a single brane is known to be described by the Born-Infeld action plus Wess-Zumino terms.

A natural question to ask is what are the next order corrections to the Born-Infeld low energy effective action. These corrections are quadratic in derivatives acting on the closed string fields. Such terms would consist of kinetic-like terms for the pull-backs of the closed string fields to the brane. For example there could be an Einstein-Hilbert 
term for the pullback of the metric or the square of the field strength for the pullback of the NS-NS two-form field. Actually though there are many other possible terms as well, eg., the square of the second fundamental term (a generalization of the extrinsic curvature for codimension $>1$ branes). It is known, for example, that a single BPS $D$-brane in Type II string theory does not have such terms for the metric [1]. This is easy to see from the string scattering amplitudes evaluated in [2, 3] for scattering a graviton off a $D$-brane. The point is simply that these amplitudes have no quadratic dependence on the momenta. The field theory terms that we are looking for however contribute to the graviton-graviton amplitude at quadratic order, and they are the only terms to contribute at this order, so one concludes that they cannot be in the low energy effective action (there are however quartic in derivative, or $\left(\alpha^{\prime}\right)^{2}$, corrections, see eg. [1, 4, 5]) .

Our purpose here is to show that this argument no longer holds for the $D$-branes appearing in the bosonic string ${ }^{1}$. Specifically we show that kinetic-like terms for the massless closed string fields are required in the $D$-brane low energy effective action in order to reproduce the string scattering amplitudes. This includes in particular the Einstein-Hilbert term for the induced metric on the brane, as well as kinetic terms for the pullbacks of the antisymmetric tensor and dilaton.

Interest in the induced Einstein-Hilbert term has appeared recently in the work of [8]. Specifically it was argued in [8] that such a term would give rise to a gravitational interaction between matter on the brane that goes like $1 / r^{p-2}$ for a $p$-dimensional brane as opposed to the naive expectation of $1 / r^{D-3}$ for a $D$ dimensional spacetime due to the bulk gravitational force. This argument presupposes that the brane is infinitely thin. It was further argued however [9] that a brane with finite thickness would also exhibit the $1 / r^{p-2}$ gravitational potential on the brane up to some crossover point, and that only for larger separations would the potential become $1 / r^{D-3}$.

The outline of the paper is as follows. In section 2 we discuss the tree level string amplitude computation corresponding to scattering a massless closed string field off a bosonic $D p$-brane. In section 3 we find the terms needed (in addition to the BornInfeld action) in the low energy effective action to reproduce these string amplitudes. In particular we find that terms quadratic in derivatives acting on the closed string fields are necessary. In section 4 we discuss ambiguities in our brane terms arising from the freedom in making field redefinitions. In section 5 we discuss the corrections these terms make to the graviton propagator, and connections with the work of [8]. In section 6 , we comment on couplings to the open-string tachyon following [10, 11].

\footnotetext{
${ }^{1}$ For the space filling $D 25$ branes see [6, 7]
} 


\section{String scattering amplitudes}

In this section we compute the tree level bosonic string scattering amplitude associated with scattering a massless closed string excitation off a $D p$-brane. The tree level computation involves computing correlators of pairs of closed string fields on the disk.

Recall that a $D p$-brane is defined in the sigma-model context as a $(p+1)$ dimensional submanifold where open strings end, eg., for a $p$-brane localized in the $x^{p+1}, \ldots, x^{D}$ directions the open string endpoints satisfy

$$
\begin{aligned}
\left.\partial_{\tau} X^{a}\right|_{\sigma=0, \pi} & =0 ; a=0, \ldots, p, \\
\left.X^{i}\right|_{\sigma=0, \pi} & =x^{i} ; i=(p+1), \ldots, D,
\end{aligned}
$$

where $D=26$ for the bosonic string. The two-point correlator of string coordinates $X^{\mu}(z, \bar{z})$ for such boundary conditions on the disk is given by

$$
\left\langle X^{\mu}(z, \bar{z}) X^{\nu}(w, \bar{w})\right\rangle=-\frac{\alpha^{\prime}}{2}\left(\eta^{\mu \nu} \ln |z-w|^{2}-V^{\mu \nu} \ln |z-\bar{w}|^{2}\right)
$$

where $\eta^{\mu \nu}=N^{\mu \nu}+D^{\mu \nu}$ and $V^{\mu \nu}=D^{\mu \nu}-N^{\mu \nu}$ with $N^{\mu \nu}=\operatorname{diag}(-1,1, \ldots, 1,0, \ldots, 0)$ where the last 1 occurs at the $p^{t h}$ diagonal index and $D^{\mu \nu}=\operatorname{diag}(0, \ldots, 0,1, \ldots, 1)$ where the first 1 occurs at the $(p+1)^{t h}$ diagonal index. The notation is chosen so that $N^{\mu \nu}$ corresponds to the metric in the Neumann boundary conditions directions and $D^{\mu \nu}$ to the metric in the Dirichlet boundary conditions directions. Note that $\eta^{\mu \nu}$ is just the Minkowski metric.

The fields that we shall be interested in are the graviton, antisymmetric tensor, and dilaton. The vertex operators for these fields are all described by (splitting the string coordinates into holomorphic and antiholomorphic pieces as $\left.X^{\mu}(z, \bar{z})=X^{\mu}(z)+\tilde{X}^{\mu}(\bar{z})\right)$

$$
V_{\epsilon}=\frac{2}{\alpha^{\prime}} \epsilon_{\mu \nu}: \partial X^{\mu}(z) e^{i k \cdot X(z)}:: \bar{\partial} \tilde{X}^{\nu}(\bar{z}) e^{i k \cdot \tilde{X}(\bar{z})}:
$$

where the colons denote separate normal ordering for the left and right moving fields. On shell the momentum and polarization tensor satisfy the massless and transverse conditions, $k^{2}=0=k^{\mu} \epsilon_{\mu \nu}$, as well as the following conditions for the respective vertex operators:

$$
\begin{aligned}
\epsilon_{\mu \nu} & =\epsilon_{\nu \mu}, \epsilon_{\mu}^{\mu}=0, \text { graviton, } \\
\epsilon_{\mu \nu} & =-\epsilon_{\nu \mu}, \text { antisymmetric tensor, } \\
\epsilon_{\mu \nu} & =\frac{1}{\sqrt{D-2}}\left(\eta_{\mu \nu}-k_{\mu} l_{\nu}-l_{\mu} k_{\nu}\right), k^{\mu} l_{\mu}=1, \quad \text { dilaton. }
\end{aligned}
$$


The amplitude associated with scattering any one of these closed string fields off a $D p$-brane (or converting one of these fields into a possibly different field) is given by

$$
\mathcal{A}_{D_{2}}\left(k_{1}, \epsilon_{1} ; k_{2}, \epsilon_{2}\right)=\left.g_{c}^{2} e^{-\lambda} \int_{0}^{1} d y\left\langle c(z) V_{\epsilon_{1}}(z, \bar{z}) c(w) \tilde{c}(\bar{w}) V_{\epsilon_{2}}(w, \bar{w})\right\rangle\right|_{z=i y, w=i},
$$

where $g_{c}$ is the closed string coupling constant, $\lambda$ the dilaton vev, and the $c$ 's are the ghost fields arising from fixing the $S L(2, R)$ symmetry of the disk. The ghost correlator, which provides the necessary gauge fixing measure, is straightforward to evaluate on the disk and is given by

$$
\langle c(z) c(w) \tilde{c}(\bar{w})\rangle=C_{D_{2}}^{g}(z-w)(z-\bar{w})(w-\bar{w})
$$

where $C_{D_{2}}^{g}$ is a functional determinant whose value (times some other factors and functional determinants) will be fixed by comparing to the Born-Infeld low energy effective action.

The remaining piece of the integrand (2.5) to be evaluated is the correlator of massless closed string vertex operators. The computation is straightforward but tedious and we find

$$
\begin{aligned}
\left\langle V_{\epsilon_{1}}(z, \bar{z}) V_{\epsilon_{2}}(w, \bar{w})\right\rangle & =i(2 \pi)^{p+1} \delta^{p+1}\left(k_{1}+k_{2}\right) C_{D_{2}}^{X}|z-w|^{2 k_{1} k_{2}}|z-\bar{w}|^{-2 k_{1} V k_{2}} \\
& \cdot|z-\bar{z}|^{-k_{1} V k_{1}}|w-\bar{w}|^{-k_{2} V k_{2}}\left[\frac{d_{1}}{|z-w|^{2}|z-\bar{w}|^{2}}\right. \\
& -\frac{1}{(z-\bar{z})(w-\bar{w})}\left(\frac{d_{2}}{|z-w|^{2}}-\frac{d_{3}}{|z-\bar{w}|^{2}}\right)+\frac{d_{4}}{|z-\bar{w}|^{4}} \\
& +\frac{d_{5}}{|z-w|^{4}}+\frac{1}{|z-\bar{z}|^{2}|w-\bar{w}|^{2}}\left(d_{6}+d_{7} \frac{|z-\bar{w}|^{4}}{|z-w|^{4}}\right. \\
& \left.\left.-d_{8} \frac{|z-\bar{w}|^{2}}{|z-w|^{2}}-d_{9} \frac{|z-w|^{2}}{|z-\bar{w}|^{2}}+d_{10} \frac{|z-w|^{4}}{|z-\bar{w}|^{4}}\right)\right]
\end{aligned}
$$

where $C_{D_{2}}^{X}$ is a functional determinant and the coefficients are given by

$$
\begin{aligned}
d_{1} & =\left[\frac{1}{2} k_{2} \epsilon_{1}^{T} \epsilon_{2} k_{1}+\frac{1}{2} k_{2} \epsilon_{1} \epsilon_{2}^{T} k_{1}-k_{2} \epsilon_{1} V \epsilon_{2} k_{1}-k_{2} \epsilon_{1}^{T} \epsilon_{2} V k_{2}-k_{2} \epsilon_{1} \epsilon_{2}^{T} V k_{2}\right. \\
& +(1 \leftrightarrow 2)] \\
d_{2} & =\operatorname{Tr}\left(\epsilon_{1} V\right)\left(k_{1} \epsilon_{2} k_{1}\right)+(1 \leftrightarrow 2) \\
d_{3} & =k_{1} V \epsilon_{1} V \epsilon_{2} V k_{2}+\frac{1}{2} k_{1} V \epsilon_{1}^{T} \epsilon_{2} V k_{2}+\frac{1}{2} k_{1} V \epsilon_{1} \epsilon_{2}^{T} V k_{2} \\
& +\operatorname{Tr}\left(\epsilon_{1} V\right)\left(k_{1} V \epsilon_{2} V k_{1}\right)+(1 \leftrightarrow 2)
\end{aligned}
$$




$$
\begin{aligned}
d_{4} & =\operatorname{Tr}\left(\epsilon_{1} V \epsilon_{2} V\right)+k_{1} V \epsilon_{2} V \epsilon_{1} V k_{2}+k_{2} V \epsilon_{1} V \epsilon_{2} V k_{1} \\
d_{5} & =\operatorname{Tr}\left(\epsilon_{1} \epsilon_{2}^{T}\right)-k_{1} \epsilon_{2}^{T} \epsilon_{1} k_{2}-k_{2} \epsilon_{1} \epsilon_{2}^{T} k_{1} \\
d_{6} & =\left[\frac{1}{2} \operatorname{Tr}\left(\epsilon_{1} V\right) \operatorname{Tr}\left(\epsilon_{2} V\right)+\operatorname{Tr}\left(\epsilon_{1} V\right)\left(k_{2} V \epsilon_{2} V k_{2}\right)+\left(k_{2} \epsilon_{1} k_{2}\right)\left(k_{1} V \epsilon_{2} V k_{1}\right)\right. \\
& +\frac{1}{2}\left(k_{2} \epsilon_{1} V k_{2}\right)\left(k_{1} \epsilon_{2} V k_{1}\right)+\left(k_{2} \epsilon_{1} V k_{2}\right)\left(k_{1} V \epsilon_{2} k_{1}\right)+\frac{1}{2}\left(k_{2} V \epsilon_{1} k_{2}\right)\left(k_{1} V \epsilon_{2} k_{1}\right) \\
& +(1 \leftrightarrow 2)] \\
d_{7} & =\left(k_{1} \epsilon_{2} k_{1}\right)\left(k_{2} \epsilon_{1} k_{2}\right) \\
d_{8} & =\left(k_{2} \epsilon_{1} k_{2}\right)\left(k_{1} \epsilon_{2} V k_{1}+k_{1} V \epsilon_{2} k_{1}\right)+(1 \leftrightarrow 2) \\
d_{9} & =\left(k_{2} V \epsilon_{1} V k_{2}\right)\left(k_{1} \epsilon_{2} V k_{1}+k_{1} V \epsilon_{2} k_{1}\right)+(1 \leftrightarrow 2) \\
d_{10} & =\left(k_{1} V \epsilon_{2} V k_{1}\right)\left(k_{2} V \epsilon_{1} V k_{2}\right) .
\end{aligned}
$$

Our notation is such that all indices are contracted with the Minkowski metric $\eta_{\mu \nu}$, eg. $k_{2} \epsilon_{1} V \epsilon_{2} k_{1}=k_{2}^{\mu} \epsilon_{1 \mu \nu} V^{\nu \lambda} \epsilon_{2 \lambda \rho} k_{1}^{\rho}$.

The scattering amplitude of massless closed string excitations is now straightforward to evaluate. One simply substitutes the ghost (2.6) and matter (2.7) correlators into the amplitude (2.5) and makes the change of variables $y=(1-\sqrt{x}) /(1+\sqrt{x})$. The resulting integrals are then just beta functions. In detail one finds

$$
\begin{gathered}
\mathcal{A}_{D_{2}}\left(k_{1}, \epsilon_{1} ; k_{2}, \epsilon_{2}\right)=\frac{i}{4} g_{c}^{2} C_{D_{2}}(2 \pi)^{p+1} \delta^{p+1}\left(k_{1}+k_{2}\right)\left(d_{1} B(-t / 2,1+2 s)+d_{2} B(-t / 2,2 s)\right. \\
-d_{3} B(1-t / 2,2 s)+d_{4} B(1-t / 2,1+2 s)+d_{5} B(-1-t / 2,1+2 s) \\
+d_{6} B(1-t / 2,-1+2 s)+d_{7} B(-1-t / 2,-1+2 s)-d_{8} B(-t / 2,-1+2 s) \\
\left.-d_{9} B(2-t / 2,-1+2 s)+d_{10} B(3-t / 2,-1+2 s)\right) \\
\approx \frac{i}{4} g_{c}^{2} C_{D_{2}}(2 \pi)^{p+1} \delta^{p+1}\left(k_{1}+k_{2}\right)\left(-\frac{2}{t} d_{1}-\left(\frac{2}{t}-\frac{1}{2 s}\right) d_{2}-\frac{1}{2 s} d_{3}\right. \\
+\left(1+\frac{t}{2}-2 s\right) d_{4}+\left(-1+4 \frac{s}{t}+\frac{t}{2}-2 s\right) d_{5}+\left(-1+\frac{t}{4 s}+\frac{t}{2}-2 s\right) d_{6} \\
\left.+2\left(-\frac{2}{t}+\frac{1}{2 s}\right) d_{7}-\left(-\frac{2}{t}+\frac{1}{2 s}\right) d_{8}+\frac{1}{2 s} d_{9}-\frac{1}{s} d_{10}+\mathcal{O}\left(k^{4}\right)\right),
\end{gathered}
$$

where we have recorded both the exact expression for the amplitude and the expansion ${ }^{2}$ to quadratic order in momenta in terms of the $t$-channel momentum transfer ${ }^{3}$ to the

\footnotetext{
${ }^{2}$ The $\left(\alpha^{\prime}\right)^{0}$ part of this expression arises from the usual Born-Infeld action, and so should match the analogous expression from the Type II superstring [3]. Our expression corrects errors in [3] that are nontrivial for antisymmetric tensor scattering. We thank Stefan Theisen and Sebastian Uhlmann for a discussion of this point.

${ }^{3}$ We take $\alpha^{\prime}=2$ for the remainder of this paper, except when it is restored explicitly.
} 
brane defined as $t=-2 k_{1} k_{2}$ and the $s$-channel momentum along the brane defined as $s=-k_{1} N k_{2}$. The overall constant $C_{D_{2}}=e^{-\lambda} C_{D_{2}}^{X} C_{D_{2}}^{g}$ will be fixed below by comparing to the field theory limit.

\section{Comparing to the field theory limit}

Given the string scattering amplitudes computed in the last section, we would now like to find the low energy effective action for the $D p$-brane that reproduces them. For the massless closed string excitations this is well known to leading order, $\mathcal{O}\left(\left(\alpha^{\prime}\right)^{0}\right)$, namely the Born-Infeld action. In Einstein frame this is given by

$$
S_{p}^{(0)}=-\tau_{p} \int d^{p+1} \sigma e^{\Phi(-1-\gamma(p+1) / 2)} \sqrt{-\operatorname{det}\left[\tilde{G}_{a b}+e^{\gamma \Phi}\left(\tilde{B}_{a b}+2 \pi \alpha^{\prime} F_{a b}\right)\right]},
$$

where the induced metric and antisymmetric tensor on the brane are defined by

$$
\tilde{G}_{a b}=G_{M N} \frac{\partial X^{M}}{\partial \sigma^{a}} \frac{\partial X^{N}}{\partial \sigma^{b}}, \quad \tilde{B}_{a b}=B_{M N} \frac{\partial X^{M}}{\partial \sigma^{a}} \frac{\partial X^{N}}{\partial \sigma^{b}},
$$

and we have defined $\gamma=-4 /(D-2)$. Our primary interest is to find the field theory terms that reproduce the $\mathcal{O}\left(\alpha^{\prime}\right)$ terms in the string scattering amplitude. Before discussing that calculation however we shall highlight the analogous $\mathcal{O}\left(\left(\alpha^{\prime}\right)^{0}\right)$ calculation as a warm-up exercise and to develop some notation.

\subsection{Graviton scattering at $\mathcal{O}\left(\left(\alpha^{\prime}\right)^{0}\right)$}

Here we sketch the comparison of the string amplitude for scattering a graviton off a $D p$-brane to the field theory amplitude at leading order in $\alpha^{\prime}$. Aside from the brane action (3.1) we shall also need parts of the bulk low energy effective action for the massless closed string excitations. In Einstein frame to zeroth order in $\alpha^{\prime}$ this is given by

$$
\begin{aligned}
S_{b u l k}^{(0)} & =\frac{1}{2 \kappa^{2}} \int d^{D} x \sqrt{-G}\left(R-\frac{1}{12} e^{2 \gamma \Phi} H_{M N P} H^{M N P}\right. \\
& \left.+\gamma \partial_{M} \Phi \partial^{M} \Phi\right)
\end{aligned}
$$

where our conventions for the Riemann tensor, etc. are

$$
\begin{aligned}
R_{M N P}{ }^{Q} & =\partial_{N} \Gamma_{M P}^{Q}-\cdots, \quad R_{M P}=R_{M N P}^{N} \\
H_{M N P} & =3 \partial_{[M} B_{N P]} .
\end{aligned}
$$

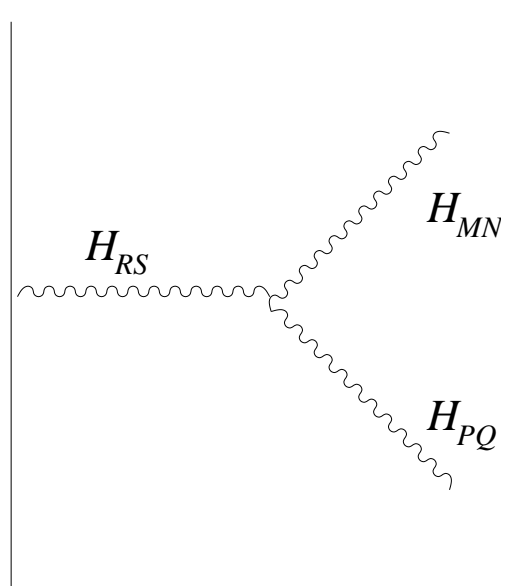

Figure 1: $t$-channel diagram for graviton-graviton scattering with graviton internal leg. 
We now have all the ingredients to check that the Born-Infeld action (3.1) correctly reproduces the string amplitude for scattering of a graviton off a $D p$-brane to leading order in $\alpha^{\prime}$. The relevant field theory diagrams for this process are displayed in figures 194. There is a $t$-channel contribution, figure 1, coming from the three point graviton vertex of $R$ in the bulk action (3.3), as well as a three point vertex involving the dilaton, figure 2, an $s$-channel contribution, figure 3, coming from the exchange of massless open string fields on the brane, and a contact contribution, figure 1 , also coming from the Born-Infeld action (3.1).

To see this in more detail we note first that to compare to the string theory amplitude we must expand the bulk (3.3) and brane (3.1) actions about Minkowski space. Furthermore we also redefine the remaining massless closed string fields so as to obtain canonically normalized kinetic terms as

$$
\begin{aligned}
G_{M N} & =\eta_{M N}+2 \kappa H_{M N}, \\
\Phi & =\kappa \sqrt{\frac{D-2}{4}} \phi, \\
B_{M N} & =-2 \kappa b_{M N} .
\end{aligned}
$$

For the bulk action this leads to the usual momentum space propagators for the graviton and dilaton,

$$
\begin{aligned}
\left\langle\tilde{H}_{M N} \tilde{H}_{P Q}\right\rangle & =-\frac{i}{2 k^{2}}\left(\eta_{M P} \eta_{N Q}+\eta_{M Q} \eta_{N P}-\frac{2}{D-2} \eta_{M N} \eta_{P Q}\right) \\
\langle\phi \phi\rangle & =-\frac{i}{k^{2}},
\end{aligned}
$$

where we are working in the de Donder gauge, $\partial^{M} H_{M N}-$ $(1 / 2) \partial_{N} H=0$, to find the graviton propagator. The three point bulk vertices following from (3.3) are similarly straightforward to write down. The only one that we shall need for graviton scattering is the three point graviton vertex coming from the EinsteinHilbert term. This can be found in eg. [12].

Expansion of the Born-Infeld action requires a few steps. First we fix the worldvolume diffeomorphism invariance by working in static gauge $X^{a}=\sigma^{a}$. The induced metric, for example, expanded about flat space, then becomes

$$
\tilde{G}_{a b}=\eta_{a b}+2 \kappa H_{a b}+2 \kappa\left(\partial_{a} X^{i} H_{i b}+H_{a j} \partial_{b} X^{j}\right)+\partial_{a} X^{i} \partial_{b} X^{j} \delta_{i j}+2 \kappa \partial_{a} X^{i} \partial_{b} X^{j} H_{i j}
$$


Next we expand the square root and determinant in (3.1) about the induced flat metric appearing in (3.7). The massless open string fields are furthermore redefined (assuming that the brane is located at $X^{i}=0$ ) as

$$
\begin{aligned}
X^{i} & =\frac{1}{\sqrt{\tau_{p}}} \lambda^{i} \\
A_{a} & =\frac{1}{2 \pi \alpha^{\prime} \sqrt{\tau_{p}}} a_{a},
\end{aligned}
$$

in order to have canonically normalized kinetic terms. Finally we note that the closed string fields evaluated on the brane are functions of the brane coordinate $X^{i}$ and should be Taylor expanded about the background configuration $X^{i}=0$. The net result is the expansion of the Born-Infeld action to quadratic order in fluctuations:

$$
\begin{aligned}
& S_{p}^{(0)}=-\tau_{p} \int d^{p+1} x\left(\frac{1}{2 \tau_{p}} \partial_{a} \lambda^{i} \partial^{a} \lambda_{i}+\frac{1}{4 \tau_{p}} f_{a b} f^{a b}+\kappa \frac{p-D / 2+2}{\sqrt{D-2}} \phi+\kappa H_{a}{ }^{a}\right. \\
& +\frac{\kappa}{\sqrt{\tau_{p}}}\left(\lambda^{i} \partial_{i} H^{a}{ }_{a}+2 \partial^{a} \lambda^{i} H_{i a}\right)+\kappa \frac{p-D / 2+2}{\sqrt{D-2}} \lambda^{i} \partial_{i} \phi+\frac{\kappa}{\sqrt{\tau_{p}}} b_{a b} f^{a b} \\
& \left.-\kappa^{2}\left(H^{a}{ }_{b} H^{b}{ }_{a}-\frac{1}{2} H_{a}^{a} H_{b}{ }_{b}\right)+\kappa^{2} \frac{p-D / 2+2}{\sqrt{D-2}} \phi H_{a}^{a}+\kappa^{2} b_{a b} b^{a b}+\cdots\right) .
\end{aligned}
$$

The momentum space $\lambda^{i}$ and $a_{a}$ propagators following from this action (working in Lorentz gauge $\partial^{a} a_{a}=0$ for the vector) are

$$
\begin{aligned}
\left\langle\tilde{\lambda}^{i} \tilde{\lambda}^{j}\right\rangle & =-\frac{i}{k^{2}} \delta^{i j}, \\
\left\langle\tilde{a}_{a} \tilde{a}_{b}\right\rangle & =-\frac{i}{k^{2}} \eta_{a b} .
\end{aligned}
$$

Now it is a simple matter to evaluate the amplitudes associated with the Feynman diagrams pictured in figures 1-4. The $t$-channel, $s$-channel, and contact term contributions are given respectively by

$$
\begin{aligned}
\mathcal{A}_{t}(h, h) & =i \frac{\tau_{p} \kappa^{2}}{t}\left(-\left(\frac{t}{2}-s\right) \operatorname{Tr}\left(\epsilon_{1} \epsilon_{2}\right)+\frac{t}{2} \operatorname{Tr}\left(\epsilon_{1} V \epsilon_{2}\right)-\operatorname{Tr}\left(\epsilon_{1} V\right)\left(k_{1} \epsilon_{2} k_{1}\right)\right. \\
& \left.-2 k_{1} \epsilon_{2} V \epsilon_{1} k_{2}+k_{1} \epsilon_{2} \epsilon_{1} k_{2}-4 k_{1} V \epsilon_{1} \epsilon_{2} k_{1}+(1 \leftrightarrow 2)\right) \\
\mathcal{A}_{s}(h, h) & =i \frac{\tau_{p} \kappa^{2}}{4 s}\left(\frac{1}{2}\left(\frac{t}{2}-s\right) \operatorname{Tr}\left(\epsilon_{1} V\right) \operatorname{Tr}\left(\epsilon_{2} V\right)+\operatorname{Tr}\left(\epsilon_{1} V\right)\left(k_{1} \epsilon_{2} k_{1}-k_{1} V \epsilon_{2} V k_{1}\right)\right. \\
& \left.-k_{1} V \epsilon_{1} \epsilon_{2} V k_{2}-k_{1} V \epsilon_{1} V \epsilon_{2} V k_{2}+(1 \leftrightarrow 2)\right) \\
\mathcal{A}_{\text {contact }}(h, h) & =i \tau_{p} \kappa^{2}\left(2 \operatorname{Tr}\left(\epsilon_{1} N \epsilon_{2} N\right)-\operatorname{Tr}\left(\epsilon_{1} N\right) \operatorname{Tr}\left(\epsilon_{2} N\right)\right) .
\end{aligned}
$$


Summing up these amplitudes one can show after some rearranging that this indeed agrees with the string amplitude (2.10) to zeroth order in $\alpha^{\prime}$ if one makes the identification

$$
\frac{1}{4} g_{c}^{2} C_{D_{2}}(2 \pi)^{p+1}=\frac{1}{2} \tau_{p} \kappa^{2}
$$

\subsection{Graviton scattering at $\mathcal{O}\left(\alpha^{\prime}\right)$}

Now we would like to construct the piece of the low energy effective action that accounts for the $\mathcal{O}\left(\alpha^{\prime}\right)$ part of the string amplitude for scattering a graviton from a $D p$-brane. The computation is exactly analogous to that presented in the previous section for the $\mathcal{O}\left(\left(\alpha^{\prime}\right)\right)$ terms. There will be $t$-channel, $s$-channel, and contact term contributions represented by the Feynman diagrams of figures 1-4. The $t$-channel contributions come from terms in the effective action that are already known, so we begin with these.

The $t$-channel contributions at quadratic order in the momenta come from bulk 3-point vertices which are quartic in derivatives and contain either three gravitons or two gravitons and a dilaton (since only the graviton and dilaton have tadpoles on the brane). Such terms have already been worked out in [13, 14] and are given by

$$
S_{R^{2}, \text { bulk }}^{(1)}=\frac{1}{2 \kappa^{2}} \int d^{D} x \sqrt{-G} \frac{\alpha^{\prime}}{4} e^{\gamma \Phi}\left(R^{M N P Q} R_{M N P Q}-4 R^{M N} R_{M N}+R^{2}\right) .
$$

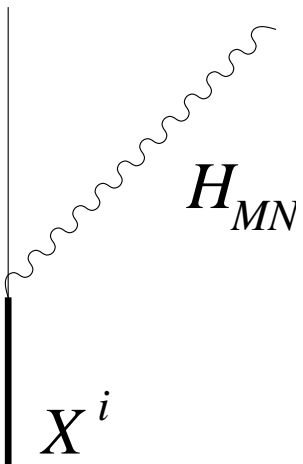

Actually only the coefficient of the square of the Riemann tensor is fixed by the string S-matrix, while the coefficients of the Ricci squared terms are arbitrary. They can be fixed by a local field redefinition (which leaves the perturbative S-matrix invariant) to the values given above. The above choice, corresponding to the

Figure 3: $s-$ channel diagram for gravitongraviton scattering with $D$-brane coordinate internal leg. Gauss-Bonnet combination, however is very convenient because the quadratic term in $H_{M N}$ appearing in it's expansion is a total derivative, and therefore there is no graviton propagator correction arising from this combination. For a detailed discussion of the ambiguities in the string effective action see [15, 14, 16].

Given this choice for the higher derivative terms in (3.14) we compute the $t$-channel amplitudes for graviton or dilaton exchange. The net result is given by

$$
\mathcal{A}_{t, R^{2}}(h, h)=i \frac{\alpha^{\prime}}{2} \tau_{p} \kappa^{2}\left(\left(\frac{t}{2}-2 s\right) \operatorname{Tr}\left(\epsilon_{1} \epsilon_{2}\right)-t \operatorname{Tr}\left(\epsilon_{1} \epsilon_{2} N\right)+2\left(1-2 \frac{s}{t}\right)\left(k_{1} \epsilon_{2} \epsilon_{1} k_{2}\right)\right.
$$




$$
\begin{aligned}
& -\frac{4}{t}\left(\left(k_{1} \epsilon_{2} k_{1}\right)\left(k_{2} N \epsilon_{1} k_{2}\right)+\left(k_{2} \epsilon_{1} k_{2}\right)\left(k_{1} N \epsilon_{2} k_{1}\right)\right)+\frac{2}{t}\left(k_{1} \epsilon_{2} k_{1}\right)\left(k_{2} \epsilon_{1} k_{2}\right) \\
& \left.-2\left(k_{1} N \epsilon_{2} \epsilon_{1} k_{2}+k_{1} \epsilon_{2} n \epsilon_{1} k_{2}+k_{1} \epsilon_{2} \epsilon_{1} N k_{2}\right)\right)
\end{aligned}
$$

This amplitude by itself already provides a check on our calculations. The only way in which to get terms proportional to $1 / t$ is via such $t$-channel amplitudes. Since the overall coefficient of the string amplitude (2.9) was already fixed in (3.13) we see that all the $1 / t$ terms in the above amplitude give us non-trivial consistency checks, which fortunately work out.

For the $s$-channel and contact term contributions we need to work a little bit harder. The Born-Infeld action clearly cannot account for these terms by itself (that is, it cannot account for the remaining terms in the string amplitude) because it gives no contribution to graviton scattering at $\mathcal{O}\left(\alpha^{\prime}\right)$. Therefore we are forced to add new terms to the brane action. The possible terms that can contribute to the amplitude at $\mathcal{O}\left(\alpha^{\prime}\right)$ come in three forms: (1) linear in $H_{M N}$ and quadratic in derivatives, (2) linear in both $H_{M N}$ and $X^{P}$ and cubic in derivatives, and (3) quadratic in both $H_{M N}$ and derivatives, all constrained of course by the symmetry requirement of diffeomorphism invariance. A term of type (1) will necessarily give rise to a term of type (2) upon expanding about the background brane configuration, but not vice versa. (1) and (2) give rise to $s$-channel contributions and (3) to contact term contributions. The most obvious term to add is the induced Ricci scalar on the brane; however, there are in fact five possible terms which satisfy this criterion. In terms of the brane action they are given by

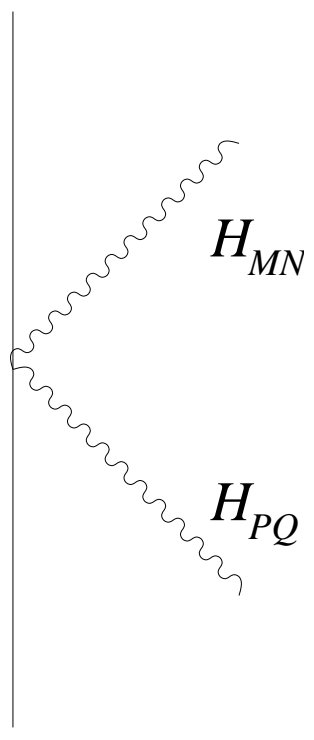

Figure 4: Contact term diagram for gravitongraviton scatter-

$$
\begin{aligned}
S_{\text {brane }}^{(1)} & =-\frac{1}{2 \kappa_{p}^{2}} \int d^{p+1} x \sqrt{-\tilde{G}}\left(\tilde{R}+\beta_{1} K_{a b}^{i} K_{i}^{a b}+\beta_{2} K_{a}^{i a} K_{i b}^{b}+\beta_{3} \perp^{M N} \stackrel{\text { ing. }}{R_{M N}}\right. \\
& \left.+\beta_{4} \perp^{M N} \perp^{P Q} R_{M P N Q}\right)
\end{aligned}
$$

where some explanation is required. We use ${ }^{\sim}$ s to denote induced quantities, so eg. $\tilde{R}$ is the Ricci scalar computed from the induced metric $\tilde{G}_{a b} . K_{a b}^{i}$ is the second fundamental form whose definition and explicit expansion we give in the appendix. The key point at the moment is that it contains terms linear in derivatives and linear in the graviton. The remaining terms are transverse projections of the bulk Riemann tensor evaluated on the brane. The projector $\perp_{M N}$ is defined in terms of the induced metric as

$$
\perp^{M N}=G^{M N}-\tilde{G}^{M N},
$$


where

$$
\tilde{G}^{M N}=\partial_{a} X^{M} \tilde{G}^{a b} \partial_{b} X^{N}
$$

These are the only ${ }^{4}$ possible terms consistent with diffeomorphism invariance (both bulk and worldbrane) and our constraint that they contribute to the contact term amplitudes at this order.

Now we fix the coefficients in (3.16) by computing all $s$-channel and contact term diagrams from the brane (3.1,3.16) action and comparing to the string amplitude. Note that the expansion of the second fundamental form as given in the appendix contains the contribution $K_{a b}^{i}=\partial_{a} \partial_{b} X^{i}+\cdots$. From the brane action (3.16) this would imply for $\beta_{2} \neq-\beta_{1}$ that there is a quadratic term for the $X^{i}$ s proportional to $\left(\beta_{1}+\beta_{2}\right)\left(\partial^{a} \partial_{a} X^{i}\right)^{2}$. Such a term would modify the propagator of the $X^{i}$ 's (or rather $\lambda^{i}$ 's after the rescaling in (3.8)). In section 1 we discuss the field redefinition ambiguities associated with the $X^{i}$ 's and show that one can use the field redefinition freedom to choose $\beta_{2}=-\beta_{1}$. Using the standard propagator for the $\lambda^{i}$ s is therefore equivalent to using the field redefinition freedom to fixing the above relation between $\beta_{1}$ and $\beta_{2}$.

Another point to note is that while the Born-Infeld action by itself does not contribute to the scattering amplitude at this order, it will contribute to the $s$-channel diagrams when combined with (3.16). Specifically the $\lambda \partial H$ type terms from (3.10) combine with the $\partial^{2} \lambda \partial H$ type terms from the expansion of the second fundamental form terms in (3.16). A lengthy calculation of the amplitudes corresponding to the diagrams displayed in figures 1-4, leads to a lengthy expression for the sum of the $s$-channel and contact term contributions at $\mathcal{O}\left(\alpha^{\prime}\right)$. The upshot is that in comparing the entire field theory amplitude (including the $t$-channel contribution as well) with the string amplitude leads to 19 equations (not including the trivial equations following from the $1 \leftrightarrow 2$ exchange symmetry of the amplitude) for the 5 coefficients. This obviously fixes the coefficients and gives many consistency checks, all of which work out fortunately. In the end we find that the coefficients (in units where $\alpha^{\prime}=2$ ) are given by

$$
\frac{1}{\kappa_{p}^{2}}=2 \tau_{p}, \quad \beta_{1}=-1, \quad \beta_{2}=1, \quad \beta_{3}=0=\beta_{4} .
$$

\subsection{Dilaton and Antisymmetric Tensor scattering}

The analysis for the dilaton and antisymmetric tensor is completely analogous. The leading order analysis in particular just amounts to checking that the Born-Infeld correctly reproduces the string scattering amplitudes to zeroth order in $\alpha^{\prime}$.

At $\mathcal{O}\left(\alpha^{\prime}\right)$ we need to add new terms to the brane action, just as for the graviton, in order to account for the string amplitude. For the dilaton the analysis is somewhat

\footnotetext{
${ }^{4}$ Another possible term would be the Ricci scalar $R$ evaluated on the brane. This term however is not independent as follows from the generalized Gauss identity.
} 
simplified in that there is no field theory $t$-channel contribution at this order as there is no bulk vertex which is quartic in derivatives and either cubic in $\phi$ 's or quadratic in $\phi$ 's and linear in the graviton, as was shown ${ }^{5}$ in [16]. The possible terms that one could add to the brane action which are quadratic in derivatives are given by ${ }^{6}$

$$
\begin{aligned}
S_{p, \Phi}^{(1)} & =-\tau_{p} \int d^{p+1} x\left(\gamma_{1} \perp^{M N}(1-(1+\gamma(p+1) / 2) \Phi) \nabla_{M} \partial_{N} \Phi+\gamma_{2} \tilde{G}^{a b} \partial_{a} \Phi \partial_{b} \Phi\right. \\
& \left.+\gamma_{3} \perp^{M N} \partial_{M} \Phi \partial_{N} \Phi\right) .
\end{aligned}
$$

Note that the relative coefficient between the two $\gamma_{1}$ terms is fixed because an undifferentiated $\Phi$ can only appear in the action through the expansion of an exponential. In particular in the string frame the brane action corresponding to tree level string processes is of the form $e^{-\Phi} \mathcal{L}(G, B, \nabla \Phi, \ldots)$. Transforming to Einstein frame one gets an overall factor of $\exp (-(1+\gamma(p+1) / 2) \Phi)$ multiplying the $\perp^{M N} \nabla_{M} \partial_{N} \Phi$ term, which after expanding results in the above relative coefficient.

If one now computes the total amplitude for dilaton scattering off the brane to quadratic order in momenta (consisting of only the $s$-channel and contact term contributions), one finds that $\gamma_{1}$ remains a free parameter. In order to fix this freedom one must compute the amplitude for converting a graviton into a dilaton. The string amplitude again follows from (2.5) by substitution of the appropriate polarization tensors. The corresponding computation on the low-energy effective action side reveals that no additional terms on top of those already added in the previous section must be added to account for the string amplitude at $\mathcal{O}\left(\alpha^{\prime}\right)$. In particular the argument in the previous paragraph fixing the overall dilaton dependence of the brane action predicts the necessary (though not sufficient) $\Phi-H_{M N}$ coupling from the induced Ricci scalar term found in the last section to correctly reproduce the string amplitude. In particular this result tells us that $\gamma_{1}=0$. The remaining $\gamma_{i}$ coefficients are then fixed to be

$$
\gamma_{2}=-\gamma_{3}=-\frac{\gamma^{2}}{2}\left((p-D / 2+2)^{2}-2(p-D / 2+2)+D-2\right)
$$

The analysis for the antisymmetric tensor is similar to that of the graviton so we shall skip the details and highlight the main points of the $\mathcal{O}\left(\alpha^{\prime}\right)$ calculation. The field theory amplitude for scattering the antisymmetric tensor off a $D p$-brane again

\footnotetext{
${ }^{5}$ More precisely, [16] showed that one can use the field redefinition freedom that leaves the S-matrix invariant to remove any such terms.

${ }^{6}$ There is one other term that one should add to this action given by $K_{a}^{a i} n_{i}^{M} \nabla_{M} \Phi$. In section 4 we show that such a term can be removed by field redefinitions that leave the S-matrix invariant.
} 
contains a $t$-channel contribution. At $\mathcal{O}\left(\alpha^{\prime}\right)$ the relevant bulk 3-point vertex arises from the following term worked out in [16],

$$
S_{b u l k, H^{2}}^{(1)}=-\frac{1}{16 \kappa^{2}} \int d^{D} x \sqrt{G} e^{3 \gamma \Phi} R^{M N P Q} H_{M N R} H_{P Q}^{R}
$$

The Born-Infeld action by itself makes no other contributions to the scattering amplitude at this order, so as before we must add terms to the brane action which are either linear in $B_{M N}$ and quadratic in derivatives, or quadratic both in derivatives and in $B_{M N}$. The possible terms are

$$
\begin{aligned}
S_{p, B}^{(1)} & =-\tau_{p} \int d^{p+1} x\left(\rho_{1} \tilde{H}_{a b c} \tilde{H}^{a b c}+\rho_{2} \perp^{M N} H_{M P R} H_{N}{ }^{P Q}+\rho_{3} \perp^{M N} \perp^{P Q} H_{M P R} H_{N Q}{ }^{R}\right. \\
& \left.+\rho_{4} \perp^{M N} \perp^{P Q} \perp^{R S} H_{M P R} H_{N Q S}+\rho_{5}\left(\tilde{B}^{a b}+2 \pi \alpha^{\prime} F^{a b}\right) \nabla^{M} H_{M a b}\right) .
\end{aligned}
$$

Working through the details of computing the $s$-channel and contact term contributions and comparing to the string amplitude results in the coefficients

$$
\begin{aligned}
\rho_{1} & =\frac{1}{12}\left(1-\frac{p+1}{D-2}\right), & \rho_{2} & =\frac{1}{4}\left(1-\frac{p+1}{D-2}\right) \\
\rho_{3} & =-\frac{1}{2}\left(1-\frac{1}{2} \frac{p+1}{D-2}\right), & \rho_{4} & =\frac{1}{4}\left(1-\frac{1}{3} \frac{p+1}{D-2}\right),
\end{aligned}
$$

while $\rho_{5}$ cannot be determined at this order because it gives vanishing contribution to this particular scattering amplitude.

\section{Field redefinition ambiguities}

We have mentioned in passing in the previous sections that the low energy effective action is ambiguous due to the freedom of making local field redefinitions [14, 16, 15]. Namely local field redefinitions that preserve the required symmetries of the action and that do not change the pole structure, leave the S-matrix invariant. We would now like to investigate to what extent the results we have obtained for the $\mathcal{O}\left(\alpha^{\prime}\right)$ terms in the brane action are fixed independently of field redefinitions.

The most general local field redefinition of the massless closed string fields that preserves diffeomorphism invariance and the property that the dilaton cannot appear undifferentiated in the action (except in the argument of an overall exponential) is 
given by [16]

$$
\begin{aligned}
\delta G_{M N} & =\alpha^{\prime}\left(b_{1} R_{M N}+b_{2} \partial_{M} \Phi \partial_{N} \Phi+e^{3 \gamma \Phi} b_{6} H_{M P Q} H_{N}{ }^{P Q}+G_{M N}\left(b_{3} R+b_{4}(\partial \Phi)^{2}\right.\right. \\
& \left.\left.+b_{5} \nabla^{M} \partial_{M} \Phi+b_{7} e^{3 \gamma \Phi} H^{2}\right)\right) \\
\delta \Phi & =\alpha^{\prime}\left(c_{1} R+c_{2}(\partial \Phi)^{2}+c_{3} \nabla^{M} \partial_{M} \Phi+c_{4} e^{3 \gamma \Phi} H^{2}\right) \\
\delta B_{M N} & =\alpha^{\prime} e^{\gamma \Phi}\left(d_{1} \nabla^{R} H_{M N R}+d_{2} H_{R M N} \nabla^{R} \Phi\right),
\end{aligned}
$$

where $\gamma=-4 /(D-2)$. Such a field redefinition was already used to fix coefficients of some of the terms in the bulk action [14, 16]. A different choice of these coefficients would in general change the $t$-channel contribution to the amplitudes computed previously, and therefore the coefficients of the brane terms that we have added. To see exactly which brane action terms are affected by these field redefinitions we need only vary the brane action with respect to (4.1). One sees immediately that the Born-Infeld part of the action will be unaffected as it is $\mathcal{O}\left(\left(\alpha^{\prime}\right)^{0}\right)$ in the closed string fields while the field redefinitions (4.1) are $\mathcal{O}\left(\alpha^{\prime}\right)$. Variation of the Born-Infeld action with respect to the field redefinitions (4.1) however will give to rise to changes in the $\mathcal{O}\left(\alpha^{\prime}\right)$ terms computed in the previous sections.

As a specific example, the gravitational terms that are produced from varying the Born-Infeld are

$$
\begin{gathered}
\delta(B . I .)=\alpha^{\prime}(B . I .)\left(( \frac { b _ { 1 } } { 2 } + \frac { p + 1 } { 2 } b _ { 3 } - ( 1 + \gamma ( p + 1 ) / 2 ) c _ { 1 } ) \left(\tilde{R}+K_{a b}^{i} K_{i}^{a b}-K_{a}^{a i} K_{b i}^{b}\right.\right. \\
\left.\left.-\perp^{M N} \perp^{P Q} R_{M P N Q}\right)+\left(\frac{b_{1}}{2}+(p+1) b_{3}-2(1+\gamma(p+1) / 2) c_{1}\right) \perp^{M N} R_{M N}\right)(4.2)
\end{gathered}
$$

after some rewriting. The first point to note is that only two of the three coefficients $b_{1}$, $b_{3}$, and $c_{1}$ are independent. This means that only two of the five possible terms that we considered in (3.16) can be set to zero by a field redefinition. In the 'scheme' in which we have used so far we have set the coefficients of the $\perp^{M N} R_{M N}$ and $\perp^{M N} \perp^{P Q} R_{M P N Q}$ terms to zero.

In an analogous way one can show that all the $\mathcal{O}\left(\alpha^{\prime}\right)$ terms in the dilaton considered in (3.20) can be removed by a field redefinition. The necessary field redefinition however would introduce $p$ (one less the brane dimension) dependence into the bulk action. Such a dependence would be a bit strange as we have no reason to expect any particular $p$ to appear. Furthermore if we have two or more branes with at least two different brane dimensions, then one could simultaneously remove the $\mathcal{O}\left(\alpha^{\prime}\right)$ terms for only those branes of a fixed dimension, and not the others. 
The same type of analysis for the antisymmetric tensor shows that of the four coefficients $b_{6}, b_{7}, c_{4}$, and $d_{1}$, only three are independent. In fact $d_{1}$ corresponds to changing the value of $\rho_{5}$ which we were unable to fix from our analysis. Two of the remaining coefficients can then be used to remove two more $\mathcal{O}\left(\alpha^{\prime}\right)$ terms in (3.23).

As for the closed string fields one may consider field redefinitions of the open string fields that leave the S-matrix invariant. In fact we have already used this freedom in an essential way in obtaining the propagator for the $\lambda^{i}$ s. As before the idea is to consider the most general field redefinition that preserves the symmetries and that does not change the pole structure of the elementary fields. As an example consider the $X^{i}$ field redefinition

$$
\delta X^{i}=e_{1} K_{a}^{a i}+e_{2} \partial^{i} \Phi
$$

This is the most general field redefinition that is linear in derivatives (one could of course consider higher derivative terms, but these would not effect the terms that are of interest to us). Acting on the Born-Infeld action this would generate the terms

$$
\delta(B . I .)=-\tau_{p} \int d^{p+1} x e^{\beta \Phi} \sqrt{-\tilde{G}}\left(-e_{1} K_{a}^{a i} K_{b i}^{b}+\left(\beta e_{1}-e_{2}\right) K_{a}^{a i} \partial_{i} \Phi+\beta e_{2}\left(\partial_{i} \Phi\right)^{2}\right)
$$

where $\beta=-(1+\gamma(p+1) / 2)$. Two of the above three terms therefore have ambiguous coefficients. As we commented on previously, we used this field redefinition freedom to set the coefficient of the $K_{a}^{a i} \partial_{i} \Phi$ term to zero and the coefficient of the $K_{a}^{a i} K_{b i}^{b}$ term, denoted $\beta_{2}$ in (3.16), equal to minus the coefficient of the $K_{a b}^{i} K_{i}^{a b}$ term, i.e., to set $\beta_{2}=-\beta_{1}$. Note that this field redefinition, combined with the above closed string field redefinition, would not allow one to remove all the induced gravitational terms on the brane that we have found.

\section{Scattering of brane-localized fields and the Einstein-Hilbert term}

The Einstein term and the extrinsic curvature terms in (3.16) on the brane were derived by analyzing the scattering of gravitons off the brane. They also affect the scattering of particles living on the brane. We may consider, for example, the $2 \rightarrow 2$ scattering of scalars $X^{i}$ associated to the transverse fluctuations. For such a scattering process, a similar analysis to the one of previous sections, can be performed. There is a string answer, which can be computed in string perturbation theory. The leading answer comes from a disc diagram with four open string vertex operators. The next diagram 
is a cylinder with the same vertex operators. The amplitude can be compared to field theory with a brane action coupled to the bulk action. Further interactions in the brane action can be deduced by requiring agreement with the string S-matrix.

Consider the scattering of $\left\langle X\left(k_{1}\right) X\left(k_{2}\right) X\left(k_{3}\right) X\left(k_{4}\right)\right\rangle$, with 1 and 2 being the incoming particles, and 3 and 4 being the outgoing particles. The leading t-channel diagram involves exchange of a graviton which propagates in the bulk. There are corrections to the $\langle X X h\rangle$ vertex coming from the new gravitational terms we have derived. These corrections affect the leading order in $g_{s}$ amplitude, but at higher orders in momenta. The new terms also affect the next to leading order in the string expansion, by changing the propagator. It is instructive to consider the form of this correction to the four point amplitude.

The leading contribution in the t-channel gives

$$
k_{1} \cdot k_{3} k_{2} \cdot k_{4} P(\zeta) \int d^{D-p-1} p \frac{e^{i \vec{p} \cdot \vec{\epsilon}}}{q^{2}+p^{2}} \delta^{p+1}\left(\sum_{i} k_{i}\right) .
$$

The momenta $k_{i}$ have components parallel to the brane only, and $P$ is a polarization dependent factor. The momenta $k_{i}$ satisfy the on-shell condition $k_{i}^{2}=0$. The momentum of the intermediate graviton is $(q+p)$, with $q=k_{1}+k_{3}$ by momentum conservation along the brane, and $p$ is the component perpendicular to the brane. The factor $e^{i q . \epsilon}$ involves a vector in the transverse directions, $\vec{\epsilon}$ which is a point splitting regulator for the integral that separates different brane-localized vertices. We can extract a long distance potential from (5.1) by performing the integral over $p$ and Fourier transforming with respect to the spatial components of $q$. This leads to a potential $\frac{1}{r^{D-3}}$ as expected for gravity in $D$ dimensions.

The next order contribution is of the form :

$g_{s} l_{s}^{D-p-1} k_{1} \cdot k_{3} k_{2} \cdot k_{4} \tilde{P}(\zeta) \int d^{D-p-1} p_{1} \int d^{D-p-1} p_{2} \frac{q^{2} e^{i\left(\vec{p}_{1} \cdot \vec{\epsilon}_{1}+\vec{p}_{2} \cdot \vec{\epsilon}_{2}\right)}}{\left(q^{2}+p_{1}^{2}\right)\left(q^{2}+p_{2}^{2}\right)} \delta^{p+1}\left(\sum_{i} k_{i}\right)$.

The power of $l_{s}$ is easily understood from dimensional analysis. Performing the integral over the transverse momenta, we get a correction which behaves lie $q^{2 D-2 p-2}$. A spatial Fourier transform gives a potential $g_{s} l_{s}^{D-p-1} / r^{2 D-p-4}$. In the case $D=5, p=3$, this form was derived as a limit of an exact propagator in [8].

The terms in (5.2) and (5.1) become comparable when the momenta exchanged are of order $1 / g_{s}^{1 /(D-p-1)} l_{s}$, corresponding to a distance scale of $g_{s}^{1 /(D-p-1)} l_{s}$. However our low energy effective description of the scattering breaks down once the momentum become of order $1 / l_{s}$. This prevents us from seeing a transition between the D-dimensional 
gravitational force law to a $(p+1)$-dimensional force law in the context of perturbative string theory.

However it is interesting to try to extrapolate our results to strong coupling to make the brane Einstein term dominate over the bulk Einstein term. Of course the detailed results cannot be trusted in this regime, but we can hope to learn something about the qualitative behavior. The relevant length scales are:

$$
\begin{array}{cc}
l_{s} & \text { string scale } \\
l_{\text {bulk }}=g_{s}^{\frac{2}{D-2}} l_{s} & \text { bulk Planck scale } \\
l_{\text {brane }}=g_{s}^{\frac{1}{p-1}} l_{s} & \text { brane Planck scale } \\
l_{t}=g_{s}^{\frac{1}{D-p-1}} l_{s} \quad \text { transition scale } .
\end{array}
$$

In particular, we should require that the longitudinal length scale $L$ be larger than $l_{s}$, $l_{\text {brane }}$, but be smaller than $l_{t}$. This can only be satisfied for $g_{s}>1$. These inequalities also lead to the condition $p>D / 2$. The marginal case $p=D / 2$ suggests that in the context of ten-dimensional string theory, the five-brane may be the most likely candidate for brane localized gravity. It would be very interesting to develop a calculational scheme for computing the low energy effective action in this case. The D5-brane has also made an appearance (although in a somewhat different manner than considered here) in the context of localizing gravity in [17, 18]. Superstring embeddings of the scenario of [8, 9] have been recently discussed in [19].

\section{Comments on tachyon terms on the brane}

It has been implicit in the above analysis that we are taking a low momentum limit, retaining only the massless fields in the bulk, and on the worldvolume of the brane. One might worry this is not a sensible limit to take in a theory with a tachyon (both in the bulk and on the brane). Starting from string field theory, we can take such a limit provided we redefine the massive fields (including the tachyon) to absorb terms linear in the massive fields [15]. For example, a tachyon coupling of the form $T X^{M} X_{M}$ leads to a tachyon equation of motion

$$
\partial^{2} T-m_{T}^{2} T=X^{M} X_{M}
$$

and $T$ cannot be integrated out directly. With a shift of the tachyon field,

$$
\tilde{T}=T-\frac{X^{M} X_{M}}{\partial^{2}-m_{T}^{2}}
$$


we can make a systematic low momentum expansion about $\tilde{T}=0$.

However, it is interesting to consider the general properties of the coupling of the open string tachyon to the massless fields [10, 11, despite the fact that such an expansion about low momentum is not systematic. In general, all the brane-localized couplings of the bulk fields will be functions of the open string tachyon. For example, at lowest order in derivatives, the Einstein term on the brane is multiplied by a function $f\left(T_{0}\right)$. We have determined, in an expansion in powers of $T_{0}$, the leading term in $\int f\left(T_{0}\right) R \sqrt{g}$. Higher terms can be determined by further comparison of S-Matrix calculations and brane action. One general property of functions like $f\left(T_{0}\right)$ follows immediately from Sen's conjectures [10]. According to these conjectures, the minimum of the tachyon potential describes the vacuum configuration without the brane. The absence of the brane implies that all brane localized couplings should vanish at the minimum of the potential. For example. the value of $f$ when $T_{0}$ is at the minimum of the open string tachyon potential should be zero. A simple way to ensure this would be to have all the brane-localized terms to be multiplied by the one function which is known to have this property, namely the tachyon potential itself. Another possibility is to have these functions be polynomials in the potential. We hope to report on further S-matrix calculations exploring these possibilities in the near future.

\section{Acknowledgments}

We would like to thank Brandon Carter, Gia Dvali, Antal Jevicki and Stefan Theisen for helpful conversations. D.L. wishes to thank the AEI Potsdam, Germany for hospitality during the completion of this work. This research is supported in part by DOE grant DE-FE0291ER40688-Task A.

\section{A. The first and second fundamental tensors}

In this appendix we summarize some useful, but perhaps less well known, facts about submanifolds. A more detailed account of the information given here can be found in [20]. Let $\Sigma$ be a $p$-dimensional submanifold of a $D$-dimensional semi-Riemannian space $\mathcal{M}$ with metric $G_{M N}$. In some local coordinate system $X^{M}$ let $\Sigma$ be parameterized by the world-volume coordinates $\sigma^{a}$ with embedding given by $X^{M}\left(\sigma^{a}\right)$. $\Sigma$ then has an induced metric given by

$$
\tilde{G}^{a b}=\frac{\partial X^{M}}{\partial \sigma^{a}} \frac{\partial X^{N}}{\partial \sigma^{b}} G_{M N}
$$


Assuming that $\tilde{G}_{a b}$ is non-degenerate, then one can define the projection operator which projects vectors onto tangent vectors to the submanifold $\Sigma$, i.e.,

$$
\tilde{G}^{M N}=\frac{\partial X^{M}}{\partial \sigma^{a}} \frac{\partial X^{N}}{\partial \sigma^{b}} \tilde{G}^{a b}
$$

which is known as the first fundamental tensor. Furthermore one can define the projection operator onto the vector space normal to $\Sigma$ by

$$
\perp^{M N}=G^{M N}-\tilde{G}^{M N} .
$$

It follows trivially that any normal to $\Sigma, n_{M}$ say, satisfies $\perp^{M N} n_{N}=n^{M}$. In particular this allows one to show that $\perp^{M N}$ may be expanded as

$$
\perp^{M N}=\sum_{i=p+1}^{D-p} n_{i}^{M} n_{i}^{N},
$$

for any orthonormal basis $n_{i}^{M}$ of normal vectors to $\Sigma$.

In the so-called static gauge, ie., coordinates in which $\Sigma$ can be parameterized locally as $X^{a}=\sigma^{a}, X^{i}=X^{i}\left(\sigma^{a}\right)$, the induced metric takes the form (3.7) when the metric is expanded about a flat background as $G_{M N}=\eta_{M N}+2 \kappa H_{M N}$. Inserting this expansion into the definition of $\perp^{M N}$ one arrives at

$$
\perp^{M N}=D^{M N}-4 \kappa D^{(M|P|} H_{P Q} N^{N) Q}-2 D_{P}^{(M \mid} \frac{\partial X^{P}}{\partial \sigma^{a}} N^{\mid N) a},
$$

for the expansion of $\perp^{M N}$ to linear order in fluctuations.

The remaining geometrical quantity that we need to introduce here is the second fundamental form. Following [21] we define it ${ }^{7}$ through the decomposition

$$
\frac{\partial X^{M}}{\partial \sigma^{a}} \nabla_{M}\left(\frac{\partial X^{N}}{\partial \sigma^{b}} \partial_{N}\right)=\tilde{\Gamma}_{a b}^{c}\left(\frac{\partial X^{N}}{\partial \sigma^{c}} \partial_{N}\right)+K_{a b}^{i} n_{i}
$$

This expression tells one how a tangent vector, $\partial_{b}=\left(\partial X^{N} / \partial \sigma^{b}\right) \partial_{N}$, changes under infinitesimal displacement along another tangent, $\partial_{a}$, to $\Sigma$. This change has components both tangent to the submanifold which defines the connection coefficients, $\tilde{\Gamma}_{a b}^{c}$, for the induced derivative operator on $\Sigma$, and components normal to the submanifold which defines the second fundamental tensor $K_{a b}^{i}$. Projecting this decomposition onto a normal vector produces the component expression

$$
K_{a b}^{i}=\left(\frac{\partial^{2} X^{N}}{\partial \sigma^{a} \partial \sigma^{b}}+\frac{\partial X^{P}}{\partial \sigma^{a}} \frac{\partial X^{Q}}{\partial \sigma^{b}} \Gamma_{P Q}^{N}\right) n_{N}^{i} .
$$

\footnotetext{
${ }^{7}$ For an equivalent, but more covariant, starting point, see [20].
} 
Working in static gauge and expanding about the Minkowski metric produces the expansion

$$
K_{a b}^{i}=\kappa\left(\partial_{a} H_{b}{ }^{i}+\partial_{b} H_{a}{ }^{i}-\partial^{i} H_{a b}\right)+\partial_{a} \partial_{b} X^{i} .
$$

Our form of the second fundamental form is related to that in [20] by

$$
K_{a b}^{i}=\frac{\partial X^{M}}{\partial \sigma^{a}} \frac{\partial X^{N}}{\partial \sigma^{b}} K_{M N}^{P} n_{P}^{i} .
$$

\section{References}

[1] C.P. Bachas, P. Bain, and M.B. Green, Curvature terms in D-brane actions and their M-theory origin, J. High Energy Phys. 9905 (1999) 011, hep-th/9903210.

[2] I. Klebanov and L. Thorlacius, The Size of p-Branes, Phys. Lett. B 371 (1996) 51, hepth/9510200].

[3] M.R. Garousi and R.C. Myers, Superstring Scattering from D-branes, Nucl. Phys. B 475 (1996) 193, hep-th/9603194.

[4] N. Wyllard, Derivative Corrections to D-brane Actions with Constant Background Fields, Nucl. Phys. B 598 (2001) 247, hep-th/0008125.

[5] A. Fotopoulos, On $\left(\alpha^{\prime}\right)^{2}$ Corrections to the D-brane Action for Nongeodesic World Volume Embeddings, , hep-th/0104146.

[6] A.A. Tseytlin, Graviton Amplitudes, Effective Action And String Generating Functional On The Disk,, Int. J. Mod. Phys. A4 (1989) 3269.

[7] O.D. Andreev, R.R. Metsaev and A.A. Tseytlin, Covariant Calculation Of The Partition Function Of The Two-Dimensional Sigma Model On Compact 2-Surfaces,, Sov. J. Nucl. Phys. 51 (1990) 359, [Yad. Fiz. 51 (1990) 564].

[8] G. Dvali, G. Gabadadze, and M. Porrati, 4-D Gravity on a Brane in 5-D Minkowski Space, Phys. Lett. B 485 (2000) 208, hep-th/0005016.

[9] G. Dvali an G. Gabadadze Gravity on a Brane in Infinite Volume Extra Space, Phys. Rev. D 63 (2001) 065007, hep-th/0008054.

[10] A. Sen, Non-BPS states and branes in string theory, hep-th/9904207.

[11] A. Sen, Supersymmetric world-volume action for non-BPS D-branes, JHEP 9910, 008 (1999), hep-th/9909062.

[12] B.S. DeWitt, Phys. Rev. 162 (1967) 1239. 
[13] B. Zweibach, Curvature Squared Terms and String Theories, Phys. Lett. B 156 (1985) 315.

[14] R.R. Metsaev and A.A. Tseytlin, Curvature Cubed Terms in String Theory Effective Actions, Phys. Lett. B 185 (1987) 52.

[15] A.A. Tseytlin, Ambiguity in the Effective Action in String Theories, Phys. Lett. B 176 (1986) 92.

[16] R.R. Metsaev and A.A. Tseytlin, Order $\alpha^{\prime}$ (Two-Loop) Equivalence of the String Equations of Motion and the $\sigma$-Model Weyl Invariance Conditions, Nucl. Phys. B 293 (1987) 385.

[17] A. Karch and L. Randall, Locally Localized Gravity, J. High Energy Phys. 0105 (2001) 008, hep-th/0011156.

[18] A. Karch and L. Randall, Localized Gravity in String Theory,, hep-th/0105108.

[19] E. Kiritsis, N. Tetradis and T.N. Tomaras, "Thick branes and 4D gravity", hepth/0106050.

[20] B. Carter, Brane Dynamics for Treatment of Cosmic Strings and Vortons, Lectures given at $2^{\text {nd }}$ Mexican School on Gravitation and Mathematical Physics, Tlaxcala, Mexico, 1-7 Dec. 1996, hep-th/9705172.

[21] A.M. Polyakov, Gauge Fields and Strings, (Hardwood Academic Pub, 1987). 